\title{
Tracking of Aircrafts Using Software Defined Radio (SDR) With An Antenna
}

\author{
H. Venkatesh Kumar ${ }^{1}$, Surabhi. G ${ }^{2}$, Neha V³, Sandesh. Y. M' ${ }^{4}$, Sagar Kumar. H. S \\ ${ }^{1}$ Professor, department of electronics and communication engineering, Nagarjuna College of \\ Engineering and Technology, Bangalore, India \\ 2,3,4,5 Department of electronics and communication engineering, Nagarjuna College of Engineering and \\ Technology, Bangalore, India
}

\section{Article Info}

Volume 8, Issue 3

Page Number : 660-665

\section{Publication Issue}

May-June-2021

\section{Article History}

Accepted : 10 June 2021

Published : 15 June 2021

\section{ABSTRACT}

Automatic Dependent Surveillance-Broadcast (ADS-B) is one in all the favoured technologies employed in air traffic surveillance. The ADS- B uses a band of $1090 \mathrm{MHz}$. ADS-B is attended with the prevailing radar-based technologies to locate aircraft. The Next Generation Air Transportation System (NGATS) conflicts can be detected and resolved by the coexistence of radar systems and ADS-B. Here we tend to track the aircraft using Software Defined Radio, hence the complexness and the value of ADS-B system implementation is drastically reduced. SDR can receive multiple numbers of aircraft information like altitude, latitude, longitude, speed, and direction in real-time and displayed by using an appropriate antenna. The usage of SDR maximizes the coverage of data with accuracy and may accomplish timely.

Keywords : Software Defined Radio, RTL1090, Antenna, Tracking

\section{INTRODUCTION}

Software Defined Radio (SDR) is the latest addition to Digital Signal Processing (DSP). It is a technology, whereby number of the physical layer functions are software-defined. The term physical layer is not restricted to OSI layers for Network architecture however is additionally extendible to the conclusion of any physical radio architecture. SDR design is consists of an RF frontend that converts the $R F$ frequency spectrum into a baseband spectrum. This is often passed to a High-Speed ADC that digitizes the baseband samples and passes them to the DSP software enforced on the computer. The
$\mathrm{RF}$ baseband data is processed by the DSP software to extract the physical data contained in it.

The air traffic controller (ATC) provides advisory service to aircraft from the ground in noncontrolled airspace. To prevent collisions, expedite the flow of air traffic are the main aim of ATC. ATC provides security to a defensive role, operated by the military in some countries. In several countries, depending on the operating range of aircraft, the services are provided to all military and private aircraft. Depending upon the class of airspace and type of aircraft, ATC releases instructions that pilots should obey. ADS-B is defined as an Automatic Dependent Surveillance-Broadcast which 
periodically broadcasts signals for tracking aircraft to determine its position by satellite navigation. The ATC on ground stations receives the information and ADS-B is a second surveillance radar. By providing the situational awareness of air traffic, the information is received by other aircraft which leads to self-separation. ADS-B does not require external input or pilot as it is automatic. It is requiring aircraft navigation system data.

The SDR is employed in ADS-B implementation for real-time air traffic monitoring system. SDR is used to provide rapid prototyping high-performance wireless communication system with integrated software solutions. SDR is a low-cost Universal Serial Bus (USB) device which receives RF radio signals. This device is designed for DVBT (Digital Video Broadcast-Terrestrial) receivers. SDR are handled in different modes, to receive any kind of signal within the range of frequency tuner operates

\section{COMPONENETS REQUIRED}

\section{RTL-SDR Dongle}

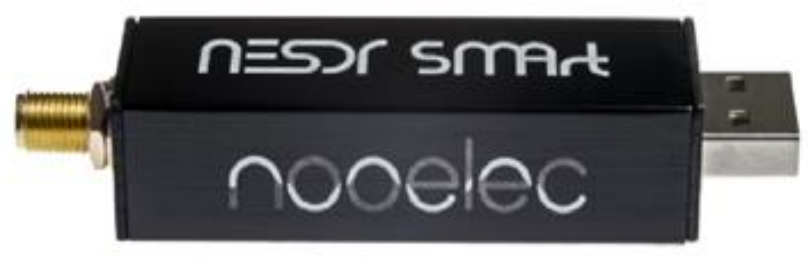

Fig 1: RTL-SDR Dongle

Fig 1: RTL-SDR is used for receiving live radio signals in a given area (no internet required). It is a low-cost USB-Dongle which is in turn computerbased radio scanner. This can receive frequencies from $500 \mathrm{kHz}$ up to $1.75 \mathrm{GHz}$ depending on the particular model. The software used for RTL-SDR is provided by license- free access in open source and is community developed.

\section{Antennas}

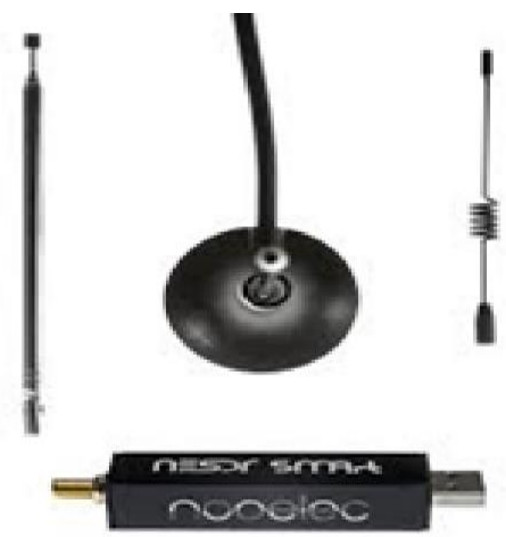

Fig 2: Antennas

Fig 2: A whip antenna is used here to track the aircraft. It is an antenna which consists of a straight flexible wire or rod. The bottom end of the whip antenna is connected to the software defined radio. It is a flexible antenna, so that it does not break easily. Whip antennas are made of series of telescoping metal tubes which can be interlocked, so that they can be retracted when not in use.

\section{Laptop}

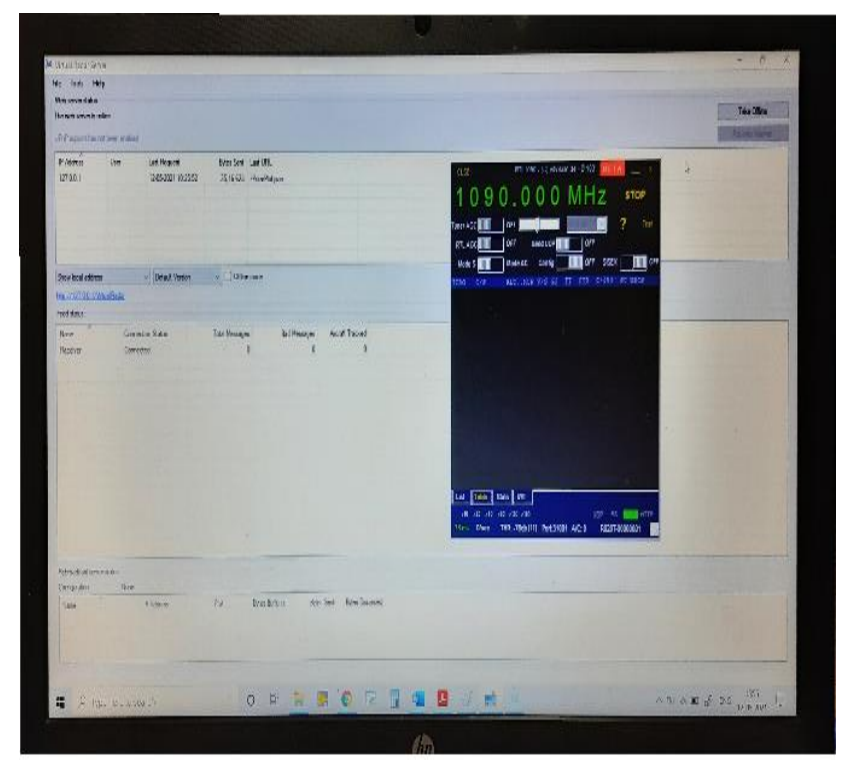

Fig 3 : Laptop 
Fig 3 : It is displaying the Laptop screen. In this project, we use the laptop to demonstrate the tracking of aircraft by installing the software.

\section{BLOCK DIAGRAM}

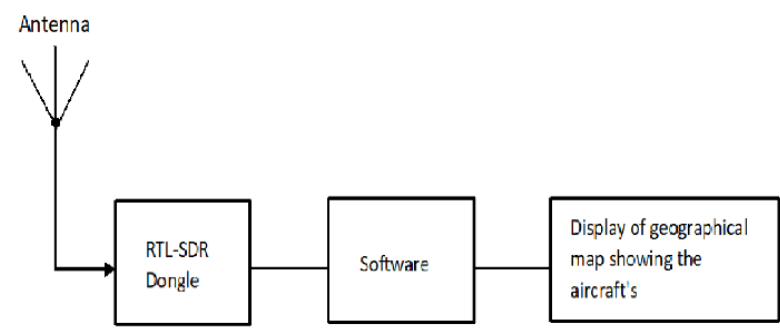

Fig 4: Block diagram of aircraft Tracking

Fig 4: In the block diagram, the antenna will be connected to the RTL-SDR dongle using a wire and the dongle will be connected to the laptop where the software has been installed. We have used software like RTL1090 to decode the signals transmitted from the ADS-B, Virtual Radar to track the aircraft using the decoder, and to display the tracked aircraft on the map. Information's like altitude, speed, distance, flight name, etc. can be acquired using this technique. The trajectory of the aircraft up to some distance will be displayed on the map.

Software-defined radio (SDR) is a system which is used for radio communication where hardware like amplifiers, filters, mixers, modulator/demodulator, etc. implemented by traditional hardware like resistors, capacitors are instead implemented by embedded systems i.e. a chipset fabricated and mounted on a PCB. The SDR used here is an RTL-SDR manufactured by RTL- SDR Blog, it consists of the RTL2832U chipset which gives us access to the radio spectrum thereby enabling us to listen to frequencies ranging from $500 \mathrm{KHz}-1.5 \mathrm{GHz}$.
RTL1090 IMU is a Windows Mode S ADS-B decoder for the RTL-SDR. To broadcast the data via TCP/IP to a graphical radar program we use this program. It simplifies the installation by automatically downloading some required .dll files. Improving decoding is the main task of the current series 2 beta version as it also has a built-in radar display.

Virtual radar is used in training aircraft, is a simulated radar system. These are not usually equipped with the radar because of cost constraints. Closed-loop data network is the basis of the virtual radar system, where the participants will be able to share their GPS positioning data and supplemental Air Data Computer (ADC) data to calculate targeting information. RTL1090 and Virtual radar are the software used in the project to track the aircraft using RTL-SDR.

\section{FLOW CHART}

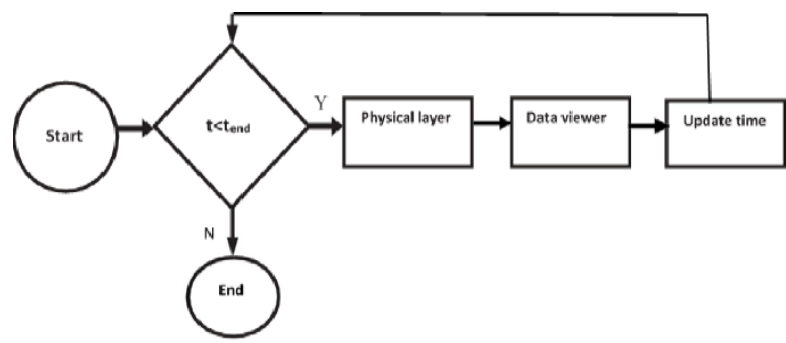

Fig 5: Implementation of ADS-B with RTL-SDR

Fig 5: At the physical layer, RTL-SDR is designed and also acts as a source. SDR samples the received signal at a rate of $2.4 \mathrm{MHz}$ with an interpolation factor of 5 to a practical sampling rate of $12 \mathrm{MHz}$ with a data rate of $1 \mathrm{Mbps}$. In the physical layer, the packet synchronizer and Mode-S transponder are present. It works on sub-frames of data equivalent to squitter packets. First the received signal with an $8-\mu$ s preamble is corelated by the synchroniser and then a peak value is determined. By checking the preamble sequence, the validation synchronization point is obtained. The received messages are shown on a Graphical User Interface 
(GUI) by the Data viewer. The time gets updated according to received messages. If received signal time $(t)$ is less than delay time (tend) then the signal gets processed to the physical layer and displays the experimental view of ADS-B. The SDR with a range of $75 \mathrm{MHz}$ to $1.25 \mathrm{GHz}$ is used in this work for tracking the information of aircraft.

\section{RESULT DISCUSSION}

The procedure of the proposed system is as follows:

Firstly, go to http://start.nesdr.com website and install the latest version of Zadig for the installation of the SDR driver for the NESDR.

Plug the NESDR into the available USB port.

Open the 'NESDR Driver Installer' named Zadig

Select the option, 'List All Devices' from the 'Options' menu in the Zadig

From the dropdown, select the NESDR called BulkIn Interface (Interface 0) and confirm whether the selected device has a USB ID of 'OBDA 2838'.

Press the 'Install Driver' to install the drivers. Now we can install the software for tracking aircraft using RTL-SDR.

Download and install open-source software named RTL1090 and Virtual Radar. It is displayed as below. Open the software and click on start present in the software where it will detect the RTL-SDR connected to the laptop and will display the tracked aircraft information like altitude, name, height, etc. under the list column.

Then open Virtual Radar software it should display the receiver as connected. Now go to Tools click on options. In the Options window select receiver in that select receiver and set the port to which SDR is connected and then click on the wizard. Select the option A Software Defined Radio -next - select the decoder as RTL1090 - Next - Select Yes option for the questioned asked - next - Click on finish.
Now click on Test Connection, it should give a message saying A connection can be made with these settings. - OK - OK.

In the main Virtual Radar window, it will display how many aircraft have been tracked and also the number of messages received.

To view the map, click on the link present in the Virtual Radar. In the maps go to Menu - Options select show current location and set the other features as required. Now It will display the tracking of the aircraft(s) with the altitude and speed.

And also, we can get additional information about the aircraft such as virtual speed, engines, altitude, heading, squawk, distance, species, wake turbulence, route, and speed along with the name and picture of the aircraft.

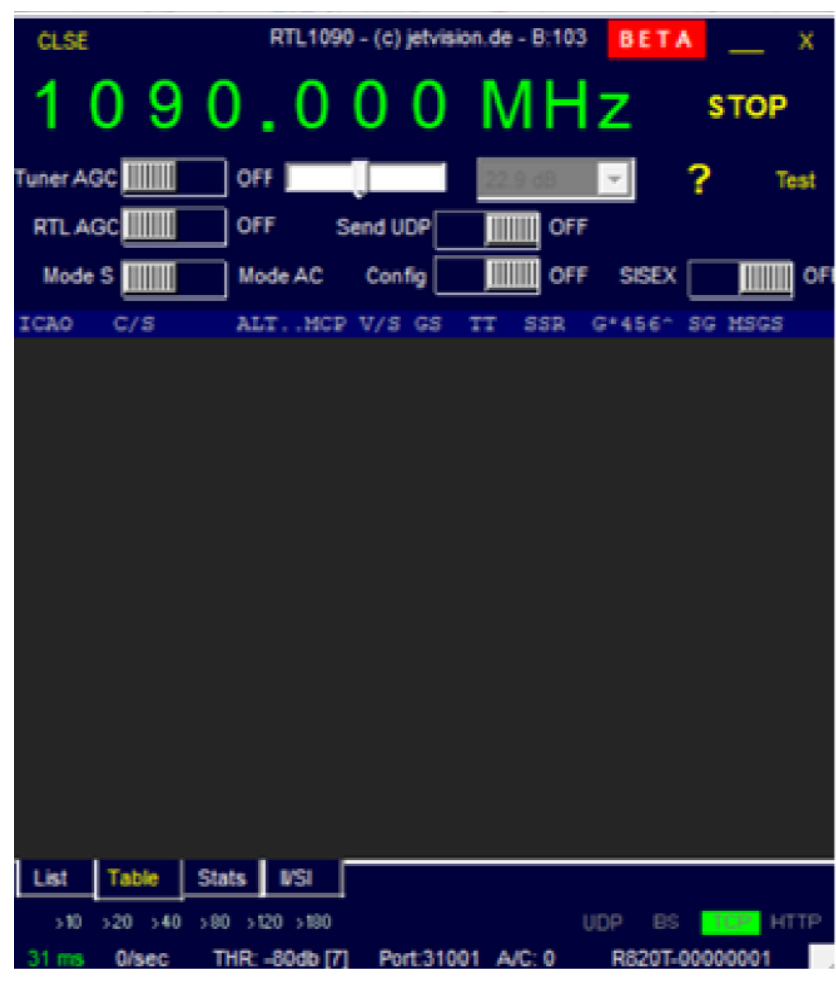

Fig 6: It displays RTL1090 Software 


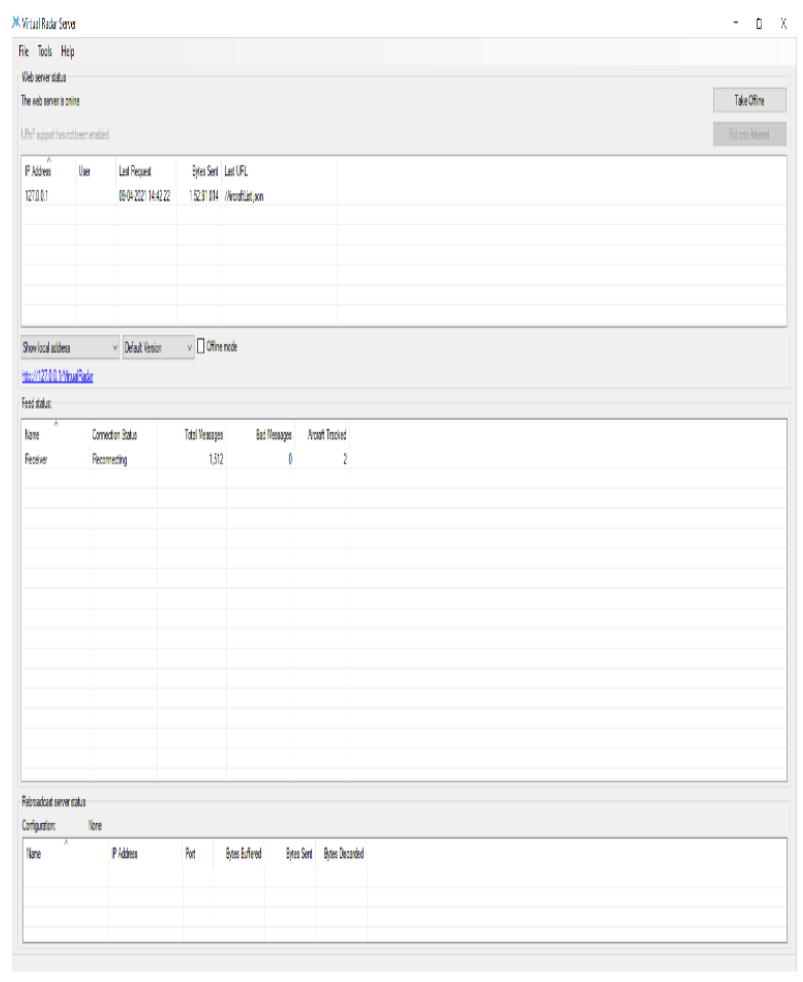

Fig 7: It displays virtual radar software

\section{SETUP FOR AIRCRAFT TRACKING}

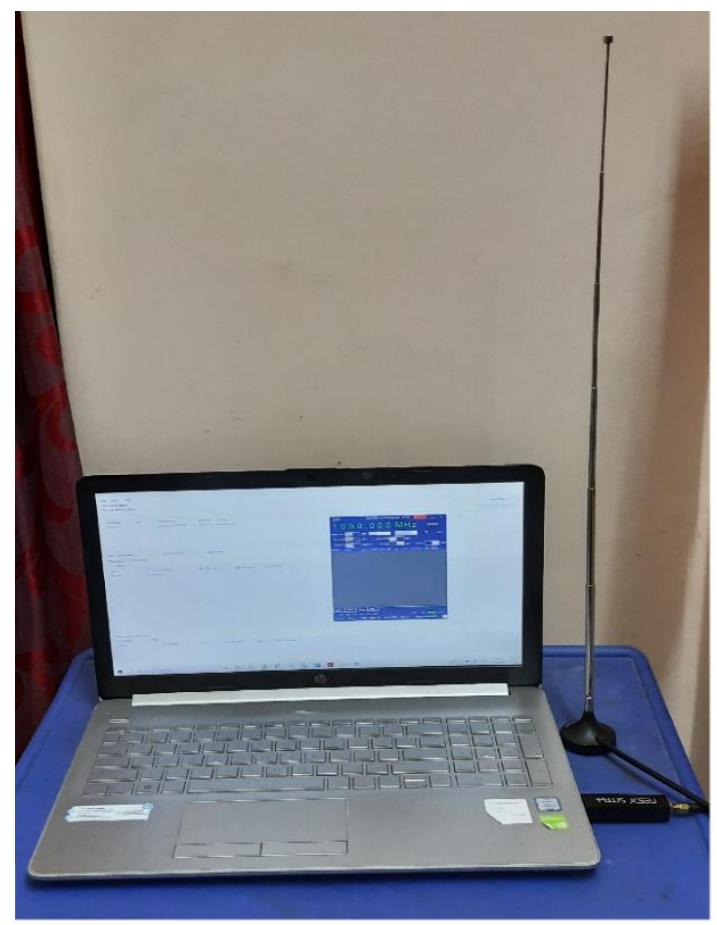

Fig 8: It displays set up of the experiment

Fig 8: The antenna is connected to the RTL-SDR dongle using a cable. The dongle is connected to the laptop where software's are installed.

\section{SIMULATION RESULTS}

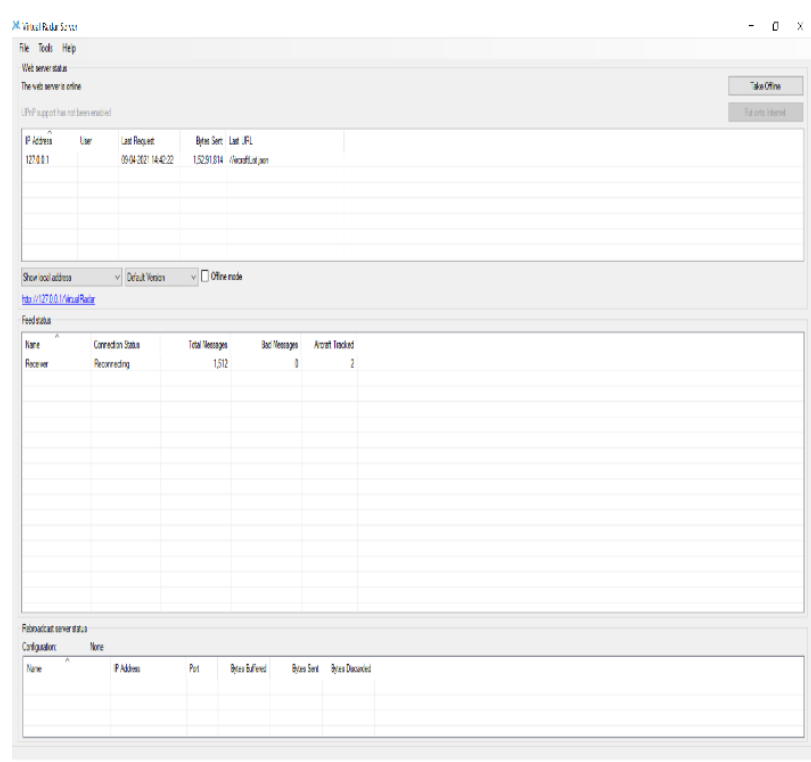

Fig 9:Displays the outcome of tracking aircraft

Fig 9: It is displaying the virtual radar software which is tracking one aircraft.

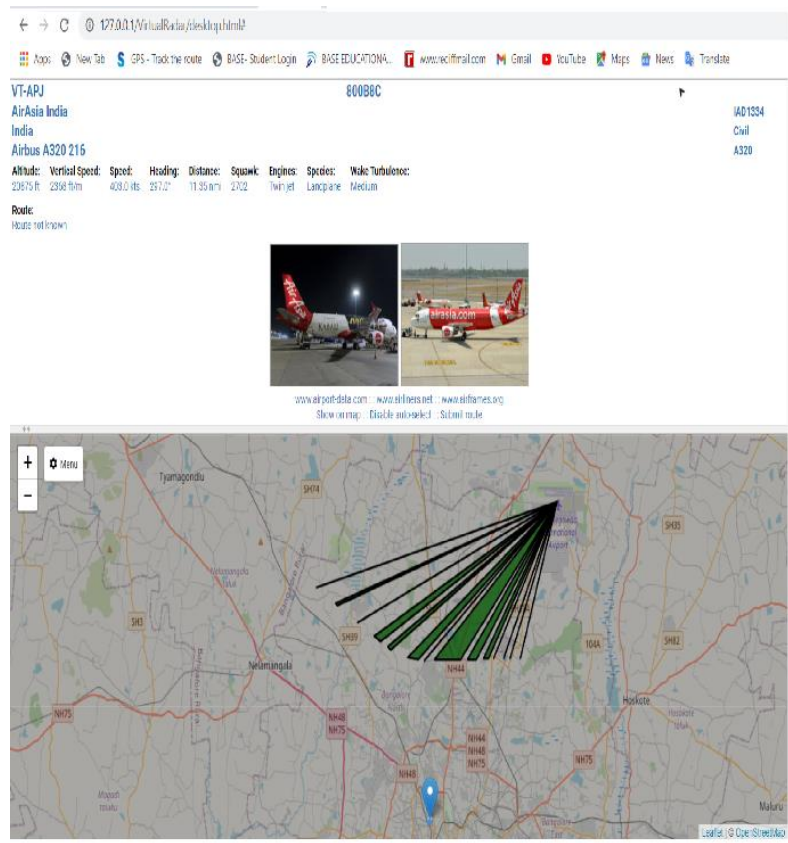

Fig 10: Displays the outcome in the map Fig10:The outcome of Aircraft Tracking has been displayed above in the map using the virtual radar software. Here we have tracked VT-SS APJ Air Asia India Airbus, which is at $20875 \mathrm{ft}$ altitude, speed of $403 \mathrm{kts}$ at the latitude-12.99750 and longitude-77.5682. 


\section{CONCLUSION}

ADS-B is the most important part of ATC for aircraft tracking systems in ATC because it provides aircraft information. To reduce the cost and complexity of the ADS-B system, the system is implemented with help of RTL-SDR. The RTL-SDR is user-friendly and easy for tracking. The SDR receives the information of Aircraft like altitude, longitude, latitude, and location are displayed. SDR is used to track the aircraft without disturbing the original aircraft broadcasting so that we can improve the efficiency of the system.

\section{REFERENCES}

[1]. W. H. W. Tuttlebee, "Software-defined radio: Facets of a developing technology", IEEE Pers. Communication., vol. 6, no. 2, pp. 38-44, Apr. 1999.

[2]. V. B. Alluri, J. R. Heath, M. Lhamon, "A new multichannel coherent amplitude-modulated time-division multiplexed software-defined radio receiver architecture and fieldprogrammable-gate-array technology implementation", IEEE Trans. Signal Process., vol. 58, no. 10, pp. 5369-5384, Oct. 2010

[3]. Akshay N, Shruthi R, Sushmitha K N, Vanitha $\mathrm{R}$, and Dr. Rekha K R, "Live Aircraft Detection with Mode-S Transponder Using RTL-SDR", International Journal of Advanced Research in Computer Science and Software Engineering, vol. 7, issue. 5, pp. 490-496, May. 2017.

[4]. Dr. Yedukondalu Kamatham and Sushmitha Pollamoni "Implementation of Automatic Aircraft Tracking with RTL-SDR", CVR Journal of Science and Technology, vol. 17, E-ISSN 2581-7957, P-ISSN 2581-3916, December. 2019.

[5]. G. Gilbert, "Historical Development of the Air Traffic Control System", IEEE Transaction on
Communications, Volume: 21, Issue: 5, pp: 364375, May 1973.

[6]. J. Mitola, "The Software Radio Architecture", IEEE Communication Magazine, Volume: 33, Issue: 5, pp: 26-38, May 1995.

[7]. Edward A Lester and R. John. Hansman, "Benefits and Incentives for ADS-B Equipage in the National Airspace System", Mass Institute of Technology, ICAT-2007.

\section{Cite this article as :}

H. Venkatesh Kumar, Surabhi. G, Neha V, Sandesh. Y. M, Sagar Kumar. H. S, " Tracking of Aircrafts Using Software Defined Radio (SDR) With An Antenna", International Journal of Scientific Research in Science and Technology(IJSRST), Print ISSN : 2395-6011, Online ISSN : 2395-602X, Volume 8, Issue 3, pp.660665, May-June-2021. Available at doi : https://doi.org/10.32628/IJSRST2183148 Journal URL : https://ijsrst.com/IJSRST2183148 